\title{
A Rational Agent Controlling an Autonomous Vehicle: Implementation and Formal Verification
}

\author{
Lucas E. R. Fernandes Vinicius Custodio Gleifer V. Alves \\ Informatics Department, UTFPR - Federal University of Technology - Paraná - Campus Ponta Grossa \\ Ponta Grossa, Brazil \\ \{lucfer, viniciuscustodio\}@alunos.utfpr.edu.br gleifer@utfpr.edu.br \\ Michael Fisher \\ Department of Computer Science, University of Liverpool, Liverpool, United Kingdom \\ MFisher@liverpool.ac.uk
}

\begin{abstract}
The development and deployment of Autonomous Vehicles (AVs) on our roads is not only realistic in the near future but can also bring significant benefits. In particular, it can potentially solve several problems relating to vehicles and traffic, for instance: (i) possible reduction of traffic congestion, with the consequence of improved fuel economy and reduced driver inactivity; (ii) possible reduction in the number of accidents, assuming that an $\mathrm{AV}$ can minimise the human errors that often cause traffic accidents; and (iii) increased ease of parking, especially when one considers the potential for shared AVs. In order to deploy an AV there are significant steps that must be completed in terms of hardware and software. As expected, software components play a key role in the complex AV system and so, at least for safety, we should assess the correctness of these components.

In this paper, we are concerned with the high-level software component(s) responsible for the decisions in an AV. We intend to model an AV capable of navigation; obstacle avoidance; obstacle selection (when a crash is unavoidable) and vehicle recovery, etc, using a rational agent. To achieve this, we have established the following stages. First, the agent plans and actions have been implemented within the GWENDOLEN agent programming language. Second, we have built a simulated automotive environment in the Java language. Third, we have formally specified some of the required agent properties through LTL formulae, which are then formally verified with the AJPF verification tool. Finally, within the MCAPL framework (which comprises all the tools used in previous stages) we have obtained formal verification of our AV agent in terms of its specific behaviours. For example, the agent plans responsible for selecting an obstacle with low potential damage, instead of a higher damage obstacle (when possible) can be formally verified within MCAPL. We must emphasise that the major goal (of our present approach) lies in the formal verification of agent plans, rather than evaluating real-world applications. For this reason we utilised a simple matrix representation concerning the environment used by our agent.
\end{abstract}

\section{Introduction}

As society advances, new technologies emerge that can provide new methods for helping people in their daily routine, as well as renewing seemingly obsolete objects via technology allowing society to retain benefits from the object while removing some if its problems. An object that is going through a renovation is the road vehicle. Such vehicles are very useful on a daily basis, allowing people to move around quickly and cheaply. However, in recent years the process of urbanisation has brought a huge amount of people into cities and, consequently, has vastly increased the population of cars on our roads [11]. This growth has resulted in significant problems, such as traffic congestion [5], increased occurrence of accidents [13], and significant additional pollution emitted by such vehicles.

L. Bulwahn, M. Kamali, S. Linker (Eds.): First Workshop on Formal Verification of Autonomous Vehicles (FVAV 2017). EPTCS 257, 2017, pp. 35 42 doi 10.4204/EPTCS.257.5 (c) L. Fernandes, V. Custodio, G. Alves \& M. Fisher

This work is licensed under the Creative Commons Attribution License. 
Autonomous vehicles represent a revolution for the automotive industry [16], not only involving new types of vehicles but also promising solutions to some of the above problems. Autonomous Vehicles, i.e: vehicles with the ability to drive themselves, will allow society to utilise the potential for road vehicles to their maximum. Usually a person has their own car, meaning that it will often only be used for a short period of time and will be left parked for the rest of the time. Cliffe [2] asserts that nowadays cars are used only $5 \%$ of the time, but with the advent of autonomous vehicles, this number is expected to increase to 75\%. According to [10], each US citizen spends around 250 (typically, unproductive) hours inside a car and $40 \%$ of the fuel on urban journeys is used while searching for parking spaces.

\subsection{Autonomy and Intelligence $\longrightarrow$ Rational Agents}

In order to develop an effective and efficient $\mathrm{AV}$, it is necessary to endow the vehicle with some form 'intelligence' that will allow it not only to perceive the world but to react to it appropriately. One way to provide this intelligence to a vehicle is through the use of intelligent agents. In the context of information systems, agents are entities that have the capacity to work independently and often, to achieve their own goals [17]. Reactive agents are devised so as to promptly respond to changes in their environment. These agents have many similarities to adaptive systems, with environmental changes being the main (sometimes only) trigger for change in behaviour. Rational agents are often more sophisticated entities, capable of taking the initiative, planning and acting autonomously, and doing so (rationally)in line with their own goals (as well as environmental constraints). In practical autonomous systems, a combination of such elements is often used. Reactive or adaptive components deal with fast environmental interaction, while a rational agent deals with the high-level 'intelligence' [8]. Such a combination has several advantages, not only in terms of combining efficient interaction with high-level reasoning (as above), but also providing a central component (the rational agent) where the decision-making and responsibility are encapsulated. This then presents the possibility of deeper analysis of the agent's choices, for example through formal verification [7].

\subsection{Reliability and Correctness $\longrightarrow$ Formal Verification}

A ratonal agent is a consistent way to endow a system with intelligence. Nevertheless, a vehicle works in critical situations that can put lives in risk. The National Highway Traffic Safety Administration (NHTSA) [12], a U.S. agency responsible for regulating autonomous vehicles, advocates that the level of intelligence of the vehicles has to surpass human driving abilities and so it is expected that the number of accidents caused by human factors drops towards zero in the era of autonomous (also known as selfdriving) cars. As mentioned in [9], the Waymo project (formerly known as Google Self-Driving Car) has decreased the number of human interventions on its road tests - these have fallen from 0.8 to 0.2 interventions per thousand miles between 2015 and 2016. As a consequence, one may infer that reduced human intervention will (likely) mean a safer AV. So, considering the deployment of an AV with its actions and decisions controlled by hardware and (particularly) software mechanisms, one may wonder: how can we guarantee that such a complex system will work reliably?

An AV has a control architecture comprising both low-level and high-level components. The lowlevel components include sensors (and actuators) for object recognition, mapping, navigation, vehicle direction, obstacle avoidance, etc [14], while the high-level components are often responsible for the decisions concerning which low-level components should be used and also when they must be activated. Assuring that a given (complex, computational) system (for example, an AV) is able to always act safely and achieve its goals thoroughly is a very hard problem. The construction of an AV requires 
several different analysis mechanisms, not only for hardware but also for software development, especially as software components can range among different layers throughout the deployment of an AV. One approach that seeks strong correctness of a system is formal verification. This involves providing a mathematical (usually logical) proof that the system matches some formal requirement. This is quite a strong commitment and, while formal verification can potentially be applied to assess many different layers within the architecture, we will primarily use it to prove that the central rational agent makes the correct decisions [7].

\subsection{Paper Overview}

We here intend to construct a rational agent in order to control the high-level decisions within an AV. As we do not construct a real $\mathrm{AV}$, we will enbed a single rational agent within an $\mathrm{AV}$ simulation environment. This set of decisions the agent can take is represented as the possible plans for the agent. In this study, the following plans have been defined: vehicle navigation; obstacle avoidance; obstacle selection (when a crash is unavoidable, the agent shall choose the obstacle with the lowest damage); and vehicle recovery (when a crash occurs).

We are concerned with verifying the choices taken by our rational agent. For instance, does our agent (based on its sensor input) always select the expected action and avoid an obstacle (when possible)?

This is the kind of question that could properly answered through the use of formal verification on the rational agent and, indeed, we will use a formal verification framework for agent programming languages called Model Checking Agent Programming Languages (MCAPL) [4]. Within MCAPL, the GWENDOLEN language [3] is used for programming the rational agent and then the Agent Java Path Finder (AJPF) model-checker is applied to these programs in order to formally verify agent properties [1]. The AJPF checker carries out an exhaustive analysis to assess whether, in all possible executions, the agent's behaviour matches it specified requirement. If not, a counter-example is generated.

The properties to be assessed represent the required actions and choices that the agent is supposed to take, depending on its beliefs. These agent beliefs are, in turn, obtained from a simulated automotive environment built in the Java language (since the MCAPL framework works with such a language). The environment comprises routes in which the agent can move towards its destination. These routes are limited (within the boundaries of the environment) and may also involve obstacles. There are three different varieties of obstacle according to the level of possible damage: low, moderate or high. With this, we can define simple formal requirements to describe AV properties, such as the one mentioned above. These formal properties are written as LTL (linear temporal logic) formulae in order to be formally verified via AJPF tool.

The reminder of the paper is organised as follows. Section 2 presents definitions concerning rational agents and formal verification. Next, in Section 3, we describe the modelling and implementation of

our agent. Section 4 shows the corresponding formal verification, while Section 5 provides concluding remarks.

\section{Rational Agents and Formal Verification}

In this section, we briefly describe some aspects used in this work. Here we use the notion of a rational agent provided by Rao and Wooldridge [18]. The authors establish that rational agents are software entities that perceive their environment through sensors. Besides, the agents have a model of their environment, and they can reason about it. Then, based on its own mental state, a rational agent can take 
actions that may change the environment [18]. A rational agent can be implemented in a number of ways, but we choose to utlise the BDI (Belief, Desire and Intention) architecture. In our work we have used a BDI programming language, named GWENDOLEN [3]. This programming language can be used with the MCAPL framework, which comprises GWENDOLEN and the linked AJPF tool [4].

AJPF is an extension of the Java Path Finder (JPF) tool, which is used for formal verification of Java programs [15]. The AJPF system [1] has been conceived to work with BDI agent programming languages, where the agent code can be formally verified against given properties. These properties are written in a Property Specification Language (PSL) which is itself based on LTL. The syntax of PSL is defined as follows. The syntax for property formulae, $\phi$, is given bellow, where: ag refers to a specific agent in the system; $f$ is a first-order atomic formula; and $\diamond$ ("eventually") and $\square$ ("always") are standard linear temporal logic operators [6].

$$
\phi::=\left|\mathrm{B}_{a g} f\right| \mathrm{G}_{a g} f\left|\mathrm{~A}_{a g} f\right| \mathrm{I}_{a g} f\left|\mathrm{ID}_{a g} f\right| \mathrm{P}(f)|\phi \vee \phi| \phi \wedge \phi|\neg \phi| \phi \mathrm{U} \phi|\phi \mathrm{R} \phi| \diamond \phi \mid \square \phi
$$

Here,

- $\mathrm{B}_{a g} f$ is true if ag believes $f$ to be true,

- $\mathrm{G}_{a g} f$ is true if $a g$ has a goal to make $f$ true,

- and so on with A representing actions, I representing intentions, ID representing the intention to take an action, and $\mathrm{P}$ representing percepts, i.e., properties that are true in the environment.

\section{Agent: Modelling and Implementation}

Our work brings a new approach for modelling a rational agent in order to behave as controller for an autonomous vehicle, where a simple driving mechanism is considered. As mentioned in Section 1, we focus on the high-level components of an AV control architecture. The agent acts as a taxi performing rides, picking up and dropping off passengers in different positions in its environment. In order to do so, the vehicle can move in four different directions, being able to avoid static and known obstacles ahead. Given a situation when a collision is unavoidable, the agent should try to ensure the least amount of physical damage to the vehicle, whenever this is possible.

Answering to passengers requests for rides and attempting to complete them is the agent's main, and initial, desire. Information about such rides is provided by the environment, which creates (randomly) a list $P$ of passengers, where $|P| \in \mathbb{N}$, and $p$ is a given passenger $(p \in P)$. Each $p$ is assigned a specific ride $r$, where $R$ is a list of rides and $r \in R$. Each $r$ has two distinct positions in the environment: starting point and destination point. The positioning in the environment is a square matrix of positions $A$ of order $n$; thus $A_{n, n} \mid n \in \mathbb{N}$. Each element in the matrix is a coordinate identified by $(X, Y) \mid(X, Y) \subset A$, where $X$ and $Y$ are, respectively, the corresponding row and column indexes of that element in $A_{n, n}$. Therefore, $X, Y \in \mathbb{N} \mid 0 \leq X, Y<n$. Furthermore, a coordinate $(X, Y)$ may have an obstacle $\theta$ contained in itself, hence $\theta \subset(X, Y)$. Since our environment is static, this statement will be true during all of the agent's activity. We highlight the agent does not have complete knowledge of its environment. It will acquire further knowledge as it explores the environment.

There are four sets of plans implemented with nine possible actions over the environment responsible for determining the agent's behaviour.

The first set of plans focusses on providing the agent abilities to perform a sequence of rides. As its first priority, the agent gathers its position in the environmental matrix by using the action localize from the first set of plans, once there are no unprocessed beliefs in the agent's belief set. Rides are 
requested from the environment by the agent using an action get_ride, which provides the coordinates of the starting and destination points of any new ride or the information that there are no passengers left; when the latter happens, the agent stops its execution.

For every new ride, the agent attempts to move the vehicle from its current location to the ride's starting point. Similarly, after that the agent proceeds to attain the destination point to drop off a passenger and conclude its ride. In both cases it is said the agent does a route, and on concluding this, it parks with an action park to allow the passenger to get in and out of the vehicle. If, before starting a ride or in any given moment within the ride, the agent believes that there is an obstacle in either the "pick up" or "drop off" location, that ride will be refused through an action refuse_ride.

The agent asks the environment the location of the destination point of its current route is, in relation to the vehicle's current coordinate. This is achieved by invoking an action compass. In order to complete the routes, the agent is able to move in four directions with an action drive, with these movements relating to the way the vehicle is maneuvering in the matrix. Therefore, a movement in a row, either increasing or decreasing a coordinate's indices, is said to be moving, respectively north and south. Meanwhile, movements in the matrix's column that increase or decrease its coordinate's indexes are denoted as, respectively, east and west.

When the agent moves to a new position in the environment, it receives information about coordinates in its surroundings, where such coordinates are strictly one movement away from the agent's current position. The information received from the environment tells the agent about the existence or not of any obstacle, as well as which direction from the agent's current location will lead to the obstacle. As previously stated, the environment is static and thus the agent will store beliefs related to obstacles during all its activity.

Each position through which the vehicle travels along a route stores information that helps the agent determine if a particular direction will be useful or not in reaching its destination. In this process, the agent gathers belief referring which directions led to a destination coordinate, as well as directions are taken to leave that position. If, during a route there is a direction $d l$ the agent moved from a coordinate $(\mathrm{X}, \mathrm{Y})$ towards a destination and an opposite direction $d 2$ that the vehicle got to the same location $(\mathrm{X}, \mathrm{Y})$, it is said that in order to reach the current destination, the agent can not move towards $d l$ from $(\mathrm{X}, \mathrm{Y})$. This is necessary to avoid duplicate movements during the same path. Thus, as it moves, the agent will generate a (partial) model of the environment where it is located and will discern which directions from its current position are invalid for completing a route, determining the failed attempts to complete a route. The situations detected are called 'known routes' and these routes should be avoidable by the agent.

During a route, if the agent finds an obstacle in the direction of its movement or known route, a set of obstacle avoidance plans is used. This set gives priority to convenient route adaptations taking the AV towards the destination of the current route. If the agent finds an obstacle while moving through rows of the location matrix and, in order to achieve its destination, the vehicle will now need to move through many columns in the future, the agent will adapt its route maneuvering towards the destination's column until one move in a row can be undertaken. Similarly, if the agent is moving between columns and it encounters an obstacle and it believes that the current destination, in the past, required movements through columns, then a maneuver in such a direction is executed until the agent can directly move towards the destination.

If no movement is possible because the destination is strictly in one direction, the agent will instead opt to move towards an available column (in case it is moving between rows) or row (in case it is moving between columns). Whenever a route adaptation fails, the agent will turn the vehicle to the opposite direction. If the vehicle is unable to adapt its route in three alternative directions, regardless of whether any of these have been previously chosen as routes, the agent will then move in that direction where 
movement is possible, when said situation is interpreted as a 'dead end'. In the last two situations, the agent will add beliefs through an action no_further_from attaching the coordinate that proved invalid to reach the destination, so the positions surrounding will recognise it as part of a 'known route'.

As discussed earlier, there are scenarios where the collision of the vehicle is unavoidable from its current position; such situations have, as a precondition, the existence of three obstacles surrounding the agent. Hence, if the vehicle moves in any of the three different directions, a crash will occur. In the case of a direction not containing an obstacle but the vehicle, the environment considers it to be temporarily obstructed and therefore, the agent has no maneuver options in which there will be no collision. Once the formal precondition can be verified as true, the environment generates a probability $T$ that, in its next move, the vehicle will collide with an obstacle; likewise, there is a probability $F$ that the agent can maneuver and avoid the obstacle, where $T, F \in \mathbb{R} \mid 0 \leqslant T, F \leqslant 1$ and $T+F=1$. This is associated with a damage level for each obstacle. The discrete possible classifications to be assigned to an obstacle are low, moderate and high, where for each of them contains a probability $L, M$ and $H$ of collision occurring, where $L, M, H \in \mathbb{R} \mid 0 \leqslant L, M, H \leqslant 1$ and $L+M+H=1$. In such situations, the agent will seek to choose to move towards an obstacle whose collision will cause the least amount of physical damage to the vehicle.

\section{Formal Verification of Agent Plans}

In accordance with the previous actions and plans defined for the agent, we have carried out formal verification by using the AJPF tool. Here we will only present the verification that represents the scenario responsible for obstacle selection, but all agent's plans have been formally verified ${ }^{1}$. We have chosen to describe verificaiton of the obstacle selection plan since it potentially has the most negative effect on the agent's desired behaviour. For instance, an undesired behaviour (when an obstacle selection takes place) may lead to severe car damage. This property ensures that the agent will choose to move to some coordinate where the damage is minimal in case of an unavoidable collision.

The property to be verified is: "During the entire agent's activity, in the occurrence of an unavoidable collision where there is at least one direction that the agent moves towards that will give a low level of damage to the vehicle, then that will be the direction chosen by the agent, and in the case when there is not a direction where the level of damage is low but there is one where a collision will be moderate, the agent will move towards that direction".

The verification of the such property is presented in Table 1 , where it is formally specified by a LT formula 2 . Note that, by using properties of GWENDOLEN and AJPF tool, it is possible to verify every single possible outcome of obstacle classification.

\section{Conclusion}

In this paper, we have presented a GWENDOLEN agent with plans and actions devised to model simple, basic high-level components of an Autonomous Vehicle. One of these components involves the handling of static obstacles, which works in a twofold manner: (i) obstacle avoidance; and (ii) obstacle selection (when a crash is unavoidable). After that, we have deployed a simple simulated environment in Java language with the corresponding scenario in order to test the plans and actions designed for our agent.

\footnotetext{
${ }^{1}$ Plans, models, and verifications are available at https://github. com/AVIA-UTFPR/SAE

${ }^{2}$ The specification of the machine used to perform this verification is as it follows: MacOS Sierra 10.12.5 on a Macbook Pro (Early 2015) with Intel Core i5 2.7 Ghz processor and 8 GB of memory RAM.
} 
Table 1: Unavoidable collisions and the choice for the least amount of damage

\begin{tabular}{|c|c|}
\hline Scenario settings & "Value \\
\hline Agent's initial Position & $(2,1)$ \\
\hline Total of rides & 1 \\
\hline Rides' Coordinates & $\{((4,1)-(4,0))\}$ \\
\hline Total of obstacles & 3 \\
\hline Obstacles' Coordinates & $\{(1,1),(2,2),(2,0)\}$ \\
\hline \multicolumn{2}{|c|}{ Formal Verification by AJPF } \\
\hline \multicolumn{2}{|c|}{ 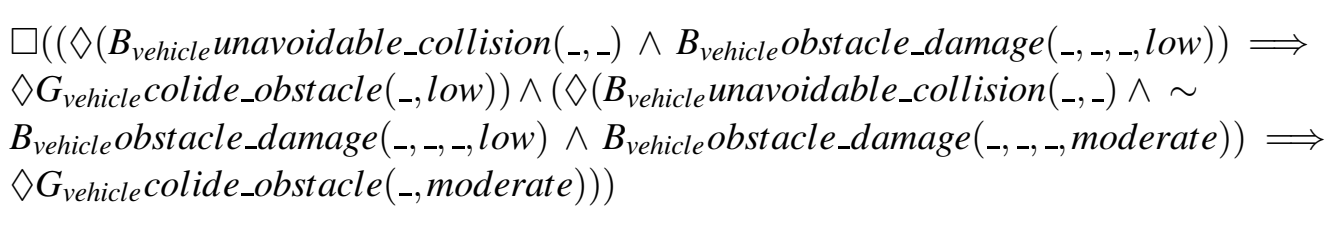 } \\
\hline Property & Feedback \\
\hline Total of environment states & 25465 \\
\hline Maximum search depth & 444 \\
\hline Total of states Büchi automata created by AJPF & 12 \\
\hline Total of run-time & 01:09:58 \\
\hline Total of RAM memory used & $3866 \mathrm{MB}$ \\
\hline
\end{tabular}

This environment represents obstacles as well as the routes traced by the agent. Next, we have chosen formal properties concerning the desired behaviour of our agent, specifically when taking into account static obstacles. These properties have been formally specified as LTL formulae and verified with the AJPF tool. As a result, we have successfully applied the MCAPL framework in order to obtain the modelling, implementation and formal verification of a simple rational agent within an AV.

This work is part of a project named Autonomous Vehicles with Intelligent Agents (AVIA). And here we present the very first results of AVIA, where we are not (yet) concerned with real world interactions. That is the reason why we have a minimal set of options and have used a (simple) matrix representation in the environment used by the rational agent. In future work, we shall extend the representation for handling multiple traffic lanes and dynamic obstacles in order to fit in more real world challenges and applications. Within this, we will define the implementation of plans and actions to avoid the dynamic obstacles. Furthermore, we aim to improve our simulated environment with the representation of lanes and roads so that we can define plans for lane crossing, lane departure and lane keeping.

Acknowledgments. The work of the last author was supported by EPSRC through its Verifiable Autonomy research project, EP/L024845.

\section{References}

[1] Rafael H. Bordini, Louise A. Dennis, Berndt. Farwer \& Michael Fisher (2008): Automated Verification of Multi-Agent Programs. In: Proceedings of the 2008 23rd IEEE/ACM International Conference on Automated Software Engineering, ASE '08, IEEE Computer Society, Washington, DC, USA, pp. 69-78. Available at http://dx.doi.org/10.1109/ASE.2008.17. 
[2] Mark Cliff (2016): Driverless cars - the route to more than smart cities. Available at https://ingworld. ing.com/en/2016-1Q/12-column-m-cliffe. Accessed, 22 May 2017.

[3] Louise A. Dennis \& Berndt Farwer (2008): Gwendolen: A BDI Language for Verifiable Agents. In: AISB Convention, University of Aberdeen, doi:10.1.1.141.1549.

[4] Louise A. Dennis, Michael Fisher, Matthew P. Webster \& Rafael H. Bordini (2012): Model Checking Agent Programming Languages. Automated Software Engineering 19(1), pp. 5-63, doi:10.1007/ s10515-011-0088-x. Available at https://doi.org/10.1007/s10515-011-0088-x.

[5] Daniel J. Fagnant \& Kara M. Kockelman (2013): Preparing a Nation for Autonomous Vehicles: Opportunities, Barriers and Policy Recommendations. Eno Center for Transportation 2.

[6] Michael Fisher (2011): An Introduction to Practical Formal Methods Using Temporal Logic. Wiley, doi:10.1002/9781119991472. Available at http://eu.wiley.com/WileyCDA/WileyTitle/ productCd-0470027886.html.

[7] Michael Fisher, Louise A. Dennis \& Matthew Webster (2013): Verifying Autonomous Systems. ACM Communications 56(9), pp. 84-93. Available at http://doi.acm.org/10.1145/2494558.

[8] Nicholas Lincoln, Sandor M. Veres, Louise A. Dennis, Michael Fisher \& Alexei Lisitsa (2010): An Agent Based Framework for Adaptive Control and Decision Making of Autonomous Vehicles. In Erdal Kayacan, editor: Proc. 10th IFAC International Workshop on the Adaptation and Learning in Control and Signal Processing, ALCOSP 2010, International Federation of Automatic Control, Istanbul, Turkey, pp. 310-317, doi:10.3182/20100826-3-TR-4015.00058. Available at https://doi.org/10.3182/ 20100826-3-TR-4015.00058.

[9] Viktor Mayer-Schonberger (2017): The last things that will make us uniquely human. Available at http://www.bbc.com/future/story/ 20170309-the-last-things-that-will-make-us-uniquely-human. Accessed, 06 June 2017.

[10] MIT Media Lab (2016): Available at https://www.media.mit.edu/. Accessed, 22 May 2017.

[11] United Nations (2015): Department of Economic and Social Affairs: Population Division. World Urbanization Prospects: The 2014 Revision. United Nations.

[12] NHTSA (2012): Preliminary Statement of Policy Concerning Automated Vehicles. Available at http:// www. autoalliance.org/index.cfm?objectid=CC9678B0-A415-11E5-997E000C296BA163. Accessed, 22 May 2017.

[13] Margie Peden, Richard Scurfield, David Sleet, Dinesh Mohan, Adnan A. Hyder, Eva Jarawan \& Colin Mathers (2004): World report on road traffic injury prevention. World Health Organization.

[14] Simon Ulbrich, Andreas Reschka, Jens Rieken, Susanne Ernst, Gerrit Bagschik, Frank Dierkes, Marcus Nolte \& Markus Maurer (2017): Towards a Functional System Architecture for Automated Vehicles. CoRR abs/1703.08557. Available at http://arxiv.org/abs/1703.08557.

[15] Willem Visser, Klaus Havelund, Guillaume P. Brat, Seungjoon Park \& Flavio Lerda (2003): Model Checking Programs. Automated Software Engineering 10(2), pp. 203-232, doi:10.1023/A :1022920129859.

[16] Richard Wallace \& Gary Silber (2012): Self-Driving cars: The next revolution. Available at https://assets.kpmg.com/content/dam/kpmg/pdf/2015/07/self-driving-cars-talkbook. pdf. Accessed, 23 May 2017.

[17] Michael Wooldridge \& Nicholas R. Jennings (1995): Intelligent Agents: Theory and Practice. Knowledge Engineering Review 10(2), pp. 115-152, doi:10.1017/S0269888900008122. Available at https://doi . org/10.1017/S0269888900008122.

[18] Michael Wooldridge \& Anand Rao (1999): Foundations of Rational Agency. Applied Logic Series 14, Springer Netherlands, doi:10.1007/978-94-015-9204-8. Available at http://www.springer.com/ gp/book/9780792356011. 